\title{
En terocolitis ileocecal aguda (tiflitis) en pacientes con neutropenia asociada a quimioterapia
}

\author{
Dra. Mireya Bravo L.' ; Dr. Juan Tordecilla C.' ; Dra. Eugenia Emparanza del S.' ; \\ Dra. Miriam Campbell B. ${ }^{\mathrm{L}}$; Dr. Jorge Vildósola S.M. ${ }^{2}$
}

\section{Acute infectious ileo-cecal inflammation (typhlitis) in neutropenic patients}

\begin{abstract}
Typhlitis, or neutropenic ileo-cecal enterocolitis (typhlitis) is an acute infectious abdominal complication observed in patients with leukemia or lymphoma during episodes of severe neutropenia related to intensive chemotherapy. Eight children followed at a metropolitan hospital of Santiago, Chile, between 1980-1986 who developed thyphlitis are presented: 4 patients had acute lymphoblastic leukemia, 2 preleukemia, one aplastic anemia and one acute myelogenous leukemia. Those under chemotherapy represented $4.6 \%$ of the patients receiving antineoplastic drugs. Abdominal pain, vomiting and intestinal bleeding were the most frequently observed symptoms. In 4 patients, cnteric bacteria were isolated from blood cultures and they were treated with broad spectrum antibiotics. Four patients were operated on because of localized peritoncal signs of an abdominal mass. Four of the 8 paticnts with typhlitis died along the course of the comptication, three were anong those surgically operated. Prompt diagnosis and aggressive treatment, with antimicrobial drugs are necessary to obtain the best results in these cases.

(Key words: cecal diseases, entetocolitis, neutropenia, agranulocytosis, granulocytopenia, leukemia, anemia, aplastic, antineoplastic agents.)
\end{abstract}

La tiflitis, enteropatía ileocecal neutropénica o enterocolitis necrotizante, del paciente neutropénico, es una entidad clínica que se ha descrito como complicación preterminal en el curso de la evolución de leucemias o linfomas y en relación con el tratamiento quimioterápico'.

Corresponde a un compromiso variado del tracto gastrointestinal, encontrándose edema, inflamación, ulceración, necrosis o infarto localizado en ciego, íleon terminal o colon ascenden$\mathrm{te}^{2,3,4}$.

Los mecanismos etiopatogénicos son variados y se ha postulado que juegan un rol importante tanto la enfermedad basal como la acción de la quimioterapia ${ }^{3}$.

Desde el punto de vista clínico y laboratorio, el diagnóstico no siempre es claro, por lo que hay que tenerlo presente y buscarlo intencionadamente ${ }^{5}$. En el último tiempo, sin embargo, el diagnóstico por imágenes ha sido de gran ayuda ${ }^{6,7}$.

Nos ha parecido de interés comunicar la experiencia clínica y terapéutica de los pacientes con el diagnóstico de tiflitis estudiados en el Hospital Roberto del Río, ya que no hemos encontrado literatura nacional al respecto. Por

1. Servicio de Pediatría, Hospital Roberto del Río. otra parte, creemos que el análisis de tres de ellos aportan elementos que ayudan a aclarar el rol, aún discutido, de los mecanismos etiopatogénicos responsables de esta complicación.

\section{MATERLAL Y METODO}

Se revisaron retrospectivamente los registros clínicos de pacientes en quienes se hizo el diagnóstico de tiflitis entre los años 1980 y 1986. Se consideró que cumplían con los requisitos para diagnosticar tiflitis aquellos pacientes que durante el curso de neutropenia presentaron fiebre, dolor abdominal y sensibilidad a la palpación, ya sea generalizada o localizada en el cuadrante inferior derecho.

Para obtenerlos se revisaron las fichas de los pacientes, que durante el período señalado recibieron quimioterapia, correspondiendo a un total de 180 niños, de éstos, 112 corresponden a leucemia linfática aguda (LLA), 43 a lencemia micloide aguda (LMA), 12 a preleucemia (PreL) y 13 a anemia aplástica (AA). En 8 niños se hizo ed diagnóstico de tiflitis de acuerdo a los requisitos anteriores. Los pacientes se enumeraron del 1 al 8 segín orden cronológico de presentación.

\section{RESULTADOS}

Los antecedentes clinicos están resumidos en la tabla 1. Nuestro material está formado por 8 pacientes de los cuales 4 eran tratados por LLA (3 en etapa de inducción y l en mantención), un 
Tabla 1

Antecedentes clínicos de 8 pacjentes con tiflitis

\begin{tabular}{|c|c|c|c|c|c|}
\hline Paciente & $\begin{array}{l}\text { Edad } \\
\text { (años) }\end{array}$ & Sexo & Diagnóstico & Droga & $\begin{array}{l}\text { Lapso entre término } \\
\text { quimioterapia y síntomas }\end{array}$ \\
\hline 1 & 5 & $\mathbf{M}$ & LLA & $\begin{array}{l}\text { Metotrexato } \\
\text { 6-Mercaptopurina }\end{array}$ & 7 días \\
\hline 2 & 10 & $\mathbf{M}$ & PreL & Citosina-arabinósido & Tratamiento en curso \\
\hline 3 & 8 & $\mathbf{M}$ & Prel & Prednisona & Tratamiento en curso \\
\hline 4 & 12 & $\mathrm{~F}$ & $A A$ & Prednisona & Tratamjento en curso \\
\hline 5 & 9 & $F$ & LLA & $V M-26$ & 10 días \\
\hline 6 & 3 & $\mathbf{M}$ & LLA & $\begin{array}{l}\text { Citosina-arabinósido } \\
\text { Adriamicina } \\
\text { Vincristina } \\
\text { L-asparaginasa } \\
\text { Prednisona }\end{array}$ & 7 días \\
\hline 7 & 11 & $\mathrm{~F}$ & LLA & $\begin{array}{l}\text { Vincristina } \\
\text { Ciclofosfamida } \\
\text { Prednisona }\end{array}$ & 7 días \\
\hline 8 & 2 & $\mathrm{~F}$ & LMA & Citosina-arabinósido & 7 dias \\
\hline
\end{tabular}

LLA: Leucemin linfática aguda; PreL: Preleucemia; AA: Anemia aplástica; LMA: Leucemia mieloide aguda.

paciente con LMA (en etapa de consolidación), 2 PreL y 1 con AA en tratamiento con prednisona.

En tres niffos (2 PreL y 1 AA) la tiflitis se presentó durante el tratamiento quimioterápico y en 5 pacientes ( 4 LLA y 1 LMA) en un período que fluctuó entre 7 a 10 dias después de suspendido.

Clínicamente destacaron el dolor abdominal presente en todos, en 5 de tipo generalizado y en 3 localizado a nivel de fosa iliaca derecha, al igual que vómitos y fiebre: en 7 hubo sangramiento digestivo y disminución de los ruidos hidroaéreos.

Desde el punto de vista de laboratorio, por definición, todos los niños tenían neutropenia marcada en el momento del diagnóstico, evolucionaron, además, con algún grado de trombocitopenia. En 4 se demostraron gérmenes en los hemocultivos (2 Pseudomona aeruginosa, $1 \mathrm{Kleb}$ siella specie y 1 Enterobacter specie).

En 4 casos el estudio radiológico de abdomen simple fue de ayuda para el diagnóstico, mostrando: gran distensión de asas intestinales en 2 niños; en otro, desplazamiento de asas hacia la izquierda del abdomen, y en el último, signos de obstrucción intestinal. A un paciente además se efectuó ecografía abdominal que evidenció un gran plastrón que ocupaba el hemiabdomen derecho.
E1 tratamiento médico se basó en el uso de antibióticos de amplio espectro en esquema triasociado (cloxacilina, gentamicina, carbenicilina); éste fue cambiado a cefotaxima y amika. cina cuando no hubo respuesta clínica o cuando los hemocultivos fueron positivos a un germen resistente al esquema en uso. En dos casos se agregó anfotericina $B$ por persistencia de la fiebre o sospecha fundada de infección por hongos a pesar de que los cultivos para éstos fueron negativos.

En todos los pacientes fue necesario transfundir hemoderivados, ya sea glóbulos rojos, sangre total o plaquetas. En dos niños fue necesario el uso de vasopresores por compromiso hemodinámico en el período preoperatorio inmediato.

Cuatro pacientes fueron intervenidos quirúrgicamente: en dos, la indicacjón estuvo dada por dolor abdominal difuso, con signos de irritación peritoneal y en los otros dos, por aparición de un plastrón en fosa ilíaca derecha. Los hallazgos quirúrgicos fueron: perforación de íleon en 1 paciente; en otro, masa en la región jleocecal con aglutinación de asas intestinales y en el que se efectuó resección de la masa y anastomosis término-terminal; en el tercero, se encontró un hematoma de la pared del ciego, efectuándose apendicectomía, y en el cuarto caso había gran edema de la pared intestinal con mucosa san. 
grante e invaginación colocecal, por lo que debió efectuarse reducción de la invaginación y apendicectomía.

En la tabla 2 se resumen las complicaciones $y$ evolución de los pacientes:

El paciente 1, con LLA, operado de una perforación de ileon, evolucionó en forma satisfactoria, siendo dado de alta. Posteriormente, continuó el tratamiento de su enfermedad basal sin incidentes, actualmente está sano y fuera de tratamiento.

Los pacientes 2, 3 y 4 fallecieron en el postoperatorio inmediato; las autopsias de los niños con PreL mostraron: necrosis masiva de intestino delgado y colon; aspergillosis y coccidiomicosis masivas, respectivamente. Los pacientes 4 y 5 fallecieron por sepsis y shock séptico. El paciente 6 , portador de una LLA, evolucionó en forma satisfactoria y continuó el tratamiento de su leucemia. El paciente 7 con LLA mejoró de su triflitis, posteriormente hizo una recaida medular y falleció. El paciente 8 , con LMA, evolucionó bien y continuó su tratamiento de la enfermedad basal, posteriormente hizo una septicemia y falleció. En sintesis, cuatro de las seis muertes estuvieron relacionadas con la tiflitis.

Tabla 2

Complicaciones y evolución de 8 pacientes con tiflitis

\begin{tabular}{|c|c|c|}
\hline Paciente & Complicaciones & Evolución \\
\hline $1 \mathrm{LLA}$ & - & $\begin{array}{l}\text { Satisfactoria. Alta. } \\
\text { Suspensión tratamiento. }\end{array}$ \\
\hline 2 PreL & Hepáticas & $\begin{array}{l}\text { Fallece. } \\
\text { Autopsia: necrosis masiva } \\
\text { de intestino delgado y colon. }\end{array}$ \\
\hline 3 PreL & Shock séptico & $\begin{array}{l}\text { Fallece. } \\
\text { Autopsia: aspergillo sis y } \\
\text { coccidiomicosis masivas. }\end{array}$ \\
\hline $4 \mathrm{AA}$ & Shock séptico & Fallece. \\
\hline 5 LLA & $\begin{array}{l}\text { Sepsis } \\
\text { Shock séptico }\end{array}$ & Fallece. \\
\hline 6 LLA & - & Satisfactoria. Alta. \\
\hline $7 \mathbf{L L A}$ & - & $\begin{array}{l}\text { Satisfactoria. Alta, } \\
\text { Recaída medular. Fallece. }\end{array}$ \\
\hline 8 LMA & Compromiso hepático séptico & $\begin{array}{l}\text { Satisfactoria; continúa trata- } \\
\text { miento. } \\
\text { Sepsis a Klebsiella. } \\
\text { Fallece. }\end{array}$ \\
\hline
\end{tabular}

\section{COMENTARIO}

En la medida que se ha introducido el uso de esquemas quimioterápicos agresivos en el manejo de los pacientes oncohematológicos, las complicaciones infecciosas han adquirido mayor importancia, tanto por su frecuencia como por su severidad, constituyéndose en la mayor causa de morbimortalidad.

La tiflitis como complicación durante el período de neutropenia intra y postquimioterapia ha sido descrita en pacientes con leucemia y linfoma; excepcionalmente, en cambio, en relación a anemia aplástica, tratamiento inmuno. supresor en trasplantes y neutropenia cíclica benigna.

En pacientes oncológicos se han reportado incidencias que varían entre $5,6 \%^{5}$ y $25 \%^{2}$ con mortalidades entre 50 a $100 \%$, concordantes con nuestra experiencia.

La etiopatogenia to está claramente establecida. Se ha postulado tanto el compromiso gastrointestinal, dado por la enfermedad basal, como el daño de la mucosa intestinal inducido por agentes quimioterápicos en un paciente neutropénico. Estos últimos, al producir ulceración de la mucosa o necrosis del tumor, podrían causar hemorragias de la mucosa o sub. 
mucosa con formación de hematomas intramurales; secundariamente se produciría invasión bacteriana a través de la mucosa, por gérmenes entéricos, to que explicaría la rápida y progresiva evolución hacia un cuadro séptico ${ }^{1,3}$.

Sin embargo, el factor predisponente más importante parece ser la necrosis de las células epiteliales de la mucosa intestinal, producida por agentes citotóxicos, que puede ser el punto de partida de infecciones y hemorragias. Apoya to anterior el hecho de encontrarse tiflitis en casos con anemia aplastica durante tratamiento con prednisona y preleucemia postquimioterapia, como sucedió en 3 de nuestros pacientes. La neutropenia prolongada, en cambio, no parece jugar igual rol, ya que no se ha descrito tiflitis en pacientes con neutropenia primaria ${ }^{4,8}$.

Si bien la tiflitis se ha descrito en relación al uso de la mayoría de los agentes quimioterápicos, los más comúnmente involucrados han sido citosina-arabinósido, agentes alquilantes, $y$ vinctistina, 6,9 . En nuestra experiencia la tiflitis se produjo en concomitancia al uso de diversos agentes quimioterápicos, siendo los más frecuentes citosina-arabinósido ${ }^{3-8}$ y corticoides $^{4-8}$.

El cuadro clínico observado en esta casuística corresponde a lo clásicamente descrito. Parece importante recalcar la relevancia de tener presente este diagnóstico toda vez que un paciente neltropénico febril presenta dolor abdominal. El reconocimiento de pacientes en riesgo y la iniciación de medidas de sostén adecuadas y agresivas son los puntos críticos para lograr sobrevida ${ }^{10}$.

Desde el punto de vista terapéutico, la conducta frente a este cuadro ha pasado por diversas etapas. Así, en las primeras publicaciones se preconizaba la cirugia precoz ${ }^{11}$, con resultados poco alentadores, debido a una alta mortalidad perioperatoria. lgual experiencia tuvimos nosotros con los primeros pacientes observados; en ellos se planteó el tratamiento quirúrgico por la gravedad de los síntomas y signos abdominales, pero que a la vez eran enfermos en muy malas condiciones generales. Posteriormente, a medida que el diaguóstico y el tratamiento médico se plantearon precozmente, vimos que era posible evitar la cirugía y obtener mejoría del cuadro clínico, como sucedió en los últimos pacientes estudiados (pacientes 6, 7,8).

Actualmente recomendamos conducta médica expectante, con observación estricta del paciente, régimen cero, hidratación y corrección de las al- teraciones hidroelectrolíticas, transfusión de hemoderivados y uso de antibióticos. El tratamiento antibiótico de amplio espectro es obligado y pretende cubrir los gérmenes que se encuentran con mayor frecuencia; para nosotros fueron Pseudomona aeruginosa, Klebsiella specie y Enterobacter specie ${ }^{12}$.

Las complicaciones médicas como sepsis, shock séptico y compromiso mutisistémico son frecuentes en la evolución de este cuadro.

La decisión quirúrgica deber ser tomada por todo el equipo médico y se plantea cuando el paciente está en condiciones adecuadas, tanto del punto de vista hematológico como hemodinámico; sin embargo, ésta deberá ser inmediata cuando persista el sangramiento gastrointestinal, una vez corregidas la trombocitopenia y las pruebas de coagulación, exista evidencia de perforación intraperitoneal o cuando se compruebe deterioro clínico progresivo del paciente con requerimiento de vasopresores o grandes cantidades de soluciones tidratantes que sugieren una sepsis incontrolable.

Nos parece, por lo tanto, que la tiflitis es una complicación grave del paciente neutropénico postquimioterapia, que debe tenerse siempre presente, toda vez que el diagnóstico oportuno ofrece mejores expectativas terapéuticas ${ }^{8}$.

\section{RESUMEN}

La tiflitis o enterocolitis neutropénica es una inflamación infecciosa aguda y fulminante, que ocurre en pacientes con leucemia o linfoma en el curso de neutropenia asociada a quimioterapia. Se presentan ocho niños seguidos en el Hospital del Roberto del Río entre 1980 y 1986 (cuatro con leucemia linfoblástica aguda, dos con preleucemia, uno con anemia aplástica y uno con leucemia mieloide aguda) que presentaron tiflitis y que corresponde a $4,6 \mathbf{4}$ de los pacientes en tratamiento quimioterápico. Clínicamente destacan el dolor abdominal, vómitos y sangramiento intestinal. En cuatro nifros encontraron gérmenes entéricos en los hemocultivos y el tratamiento médico se basó en antibióticos de amplio espectro. Cuatro niños fueron intervenidos quirúrgicamente por signos de irritación peritoneal o por presencia de masa aplastronada y cuatro fallecieron a causa de tiflitis. Se destaca la importancia de un diagnóstico y tratamiento médico precoz para lograr éxito terapéutico. 


\section{REFERENCIAS}

1. Shamberger R.C., Weinstein H.J., Delorey M.J.: The medical and surgical management of typhlitis in children with acute nonlymphocytic (mycloBenous) leukemia. Cancer 1986; 57: 603-609.

2. Prolla J.C., Kirsner J.B.: The gastrointestinal lesions and complications of the leukemias. Ann Intern Med 1964; 61: 1084-1099.

3. Amromin G.O., Solomon R.D.: Necrotizing enteropathy. A complication of treated leukemia or ly mphoma patients. JAMA 1962; 182:23-29.

4. Starnes H.F., Moore F.D., Mentzer et al. Abdominal pain in neutropenic cancer patients. Cancer $1975 ; 35: 616-621$.

5. Exalby P.R., Ghandchi $A .$, Lansigan $N_{\text {., Schwartz }}$ I. Management of the acute abdomen in children with leukemia. Cancer 1975; 35: 826-829.

6. Wagner M. Rosenberg H., Fernbach D., Singieton
E.: Typhlitis: A complication of leukemia in childhood. A.J $1970 ; 109$ : 341-350.

7. Del Fay R.L., Cronin T.G. Jr.: Typhlitis complicating leukemia in an adult: Parium cnema findings. AJR 1977; 129: 347-348.

8. Pokomey B.H., Jones J.M., Shaikh B.S. et al.i Typhlitis: Atreatable cau se of recurrent septicemia. JAMA 1980; 243; 682-683.

9. Kingry R.L., Hobson R.W., Muir R.W.: Cecal necrosis and perforation with systemic chemotherapy. Am Surg 1973; 39 : 129-133.

10. Steinberg D., Gold J., Brodin A.: Nectotizing enterocolitis in leukemia. Arch Intern Med 1973; 131: 538-544.

11. Varki A.P., Armitage J.O., Feagler J.R.: Typhilis in acute leukemia: Successful treatnent by early surgical intervention. Cancer 1979; 43: 695-697.

12. Pizzo P.A.* Grantlocy topenia and cancer therapy. Cancet 1984 ; $54: 2649-2661$. 